\title{
Effect of Indenter-Radius Size on Au(001) Nanoindentation
}

\author{
J. Knap and M. Ortiz \\ Division of Engineering and Applied Science, California Institute of Technology, Pasadena, California 91125, USA
}

(Received 3 November 2002; published 3 June 2003)

\begin{abstract}
We address the question of whether results obtained for small indenters scale to indenter sizes in the experimental range. The quasicontinuum method is used in order to extend the computational cell size to $2 \times 2 \times 1 \mu \mathrm{m}^{3}$, nominally containing of order $2.5 \times 10^{11}$ atoms, and in order to permit consideration of indenter radii in the range $70-700 \AA$. The dislocation structures for the large indenter are found to be less sharp and to extend over a larger region than for the small indenter. In addition, the large-indenter force-displacement curve differs from that corresponding to the small indenter in one important respect, namely, the absence of force drops during indentation, despite profuse dislocation activity. Based on these observations, we conclude that the indenter force is not a reliable indicator of the onset of dislocation activity and plastic deformation for indenter sizes in the experimental range.
\end{abstract}

DOI: $10.1103 /$ PhysRevLett.90.226102

PACS numbers: 02.70.-c, 31.15.-p, 46.15.-x

The objective of this work is to ascertain the effect of indenter radius on the mechanics of nanoindentation of ductile fcc crystals at zero temperature (see, e.g., [1-9] for experimental background). For definiteness, we specifically focus on the indentation of $\mathrm{Au}(001)$ by spherical indenters of tip radii 70 and $700 \AA$. One reason why the tip radius size is of concern is that straight molecular dynamics calculations have often relied on artificially small indenters in order to reduce the size of the computational cell [10-20], and the scaling of the results to larger indenters is not straightforward. In particular, it is not immediately clear how dislocation activity depends on and how effective properties such as the force vs depthof-indentation relation scales with - indenter size.

The technique we use in order to sidestep the size restrictions of straight molecular dynamics is the quasicontinuum method of Tadmor and co-workers [21-23]. Thus, by coarsening the level of spatial resolution of the computational mesh away from the indenter we are able to span realistic material samples of the order of $2 \times 2 \times$ $1 \mu \mathrm{m}^{3}$, which nominally contain $2.5 \times 10^{11}$ atoms. This helps to capture the elastic field of the indenter without introducing spurious or parasitic effects associated with periodicity or small sample sizes. By adapting the mesh size to the deformation field, the calculations provide full atomistic resolution over an appropriate region under the indenter. In this manner, the calculation is reduced to a coarse-grained system of size several orders of magnitude smaller than the original one, without appreciable loss of accuracy. In addition, this model reduction enables us to consider a wide range of indenters (70 to $700 \AA$ in the work presented here).

The particular implementation of the quasicontinuum method used in the calculations has been described in [23]. In particular, following [21-23] we adopt as adaption indicator

$$
\epsilon(K) \equiv \sqrt{\left|I I_{E}(K)\right|} \frac{h(K)}{b},
$$

where $K$ denotes a simplex in the triangulation, $I I_{E}(K)$ denotes the second invariant of the Lagrangian strain tensor in the simplex $K, h(K)$ is the size of $K$, and $b$ is the Burgers vector magnitude. It follows from its definition that $\epsilon(K)$ is invariant under rotations. The simplex $K$ is targeted for refinement if

$$
\epsilon(K)>\mathrm{TOL}
$$

for a prescribed tolerance TOL $=2 \times 10^{-3}$. The effect of the choice of tolerance on the accuracy of the method has been systematically investigated in [23]. The tolerance used in the present calculations ensures that the mesh resolution is fully atomistic well in advance of the nucleation or passage of a dislocation.

The material under consideration is fcc gold. The computational cell adopted in calculations is $2 \times 2 \times$ $1 \mu \mathrm{m}^{3}$ and oriented along the cube directions (Fig. 1). The cell encompasses approximately $0.24 \times 10^{12}$ atoms.

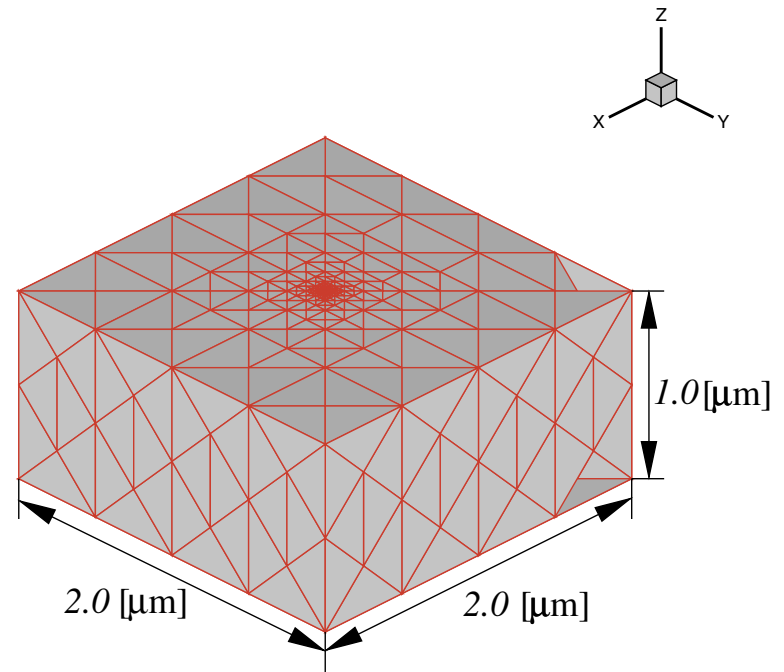

FIG. 1 (color online). Geometry of the computational cell and initial mesh. 
The bottom surface of the cell rests on a rigid half-space, whereas all other surfaces are traction-free. The energy of the crystal is modeled using the embedded-atom method potential of Johnson et al. [24,25]. Following Kelchner and co-workers $[13,20]$, we model the indenter as an additional external potential of the form $A H(R-r)(R-r)^{3}$, where $R$ is the indenter radius, $r$ denotes the distance between an atom and the center of the indenter, $A=$ $5.3 \mathrm{nN} / \AA^{2}$ is the force constant, and $H(r)$ is the step function. Two values of indenter radius are considered: 70 and $700 \AA$. The former radius represents a typical value used in previous atomistic simulations [13,20], whereas the latter radius corresponds to the nanoindentation experiments of Kiely and Houston [3].

The initial triangulation of the cube is specifically tailored to the nanoindentation geometry (Fig. 1). Thus, in a small region of the crystal located directly underneath the indenter, full atomistic resolution is supplied from the outset. Away from this region, the triangulation becomes gradually coarser. The resulting number of representative atoms in the initial mesh is 25329 , which represents a 7 order-of-magnitude reduction in the size of the calculation relative to direct atomistic simulation. The indenter is driven into the slab in small displacement increments, and at each step the new stable equilibrium configuration is computed using the Polak-Ribiere variant of the conjugate gradient method [26].

In order to reliably identify the defects in the crystal, we resort to the technique of Kelchner et al. [13], which relies on the value of the centrosymmetry parameter in order to detect and identify lattice defects. The centrosymmetry parameter is defined as

$$
P=\sum_{i=1}^{6}\left|\boldsymbol{R}_{i}+\boldsymbol{R}_{-i}\right|^{2}
$$

where $\boldsymbol{R}_{i}$ and $\boldsymbol{R}_{-i}$ are the vectors corresponding to the six pairs of opposite nearest neighbors in the fcc lattice. By way of illustration, the centrosymmetry parameter takes the value of zero for an atom in the perfect Au lattice, $24.9 \AA$ for a surface atom, $8.3 \AA$ for an atom in a stacking fault, and $2.1 \AA$ for an atom at the core of a partial dislocation. In all subsequent dislocation structure plots, the atoms are colored according to the magnitude of the centrosymmetry parameter with blue corresponding to surfaces, red to partial dislocations, and yellow to stacking faults.

The computed dislocation structures for a $70 \AA$ A-radius indenter at a depth of indentation of $9.2 \AA$ are shown in Fig. 2. As expected, slip occurs predominantly on $\{111\}$ planes, the dominant slip planes in fcc crystals. In particular, slip is observed on four sets of distinct $\{111\}$ slip planes that terminate at the (001) surface. After a certain amount of slip, dislocation loops gliding in these planes react to form locks and arrest. Further indentation then induces activity on neighboring slip planes which carry

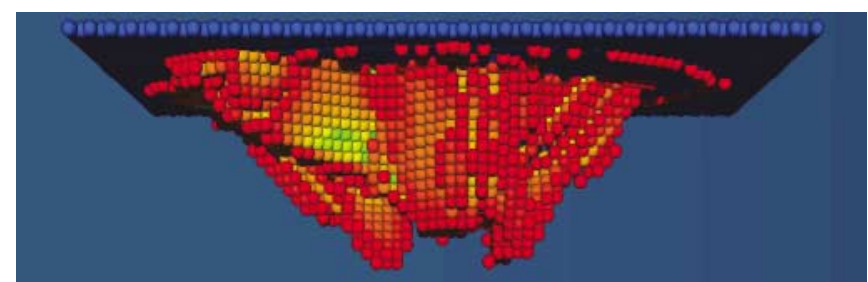

FIG. 2 (color). Dislocation structures for the $70 \AA$ A indenter at an indentation depth of $9.2 \AA$.

all the plastic deformation until they, too, become inactive, the entire process repeating itself several times in the course of the calculation.

The deformed computational mesh at a depth of penetration of $9.2 \AA$ is shown in Fig. 3. The total number of representative atoms in this configuration is 203816, which represents almost an order of magnitude increase with respect to the initial triangulation. All new representative atoms are inserted in the vicinity of the indenter, with the result that the induced dislocation structures are contained in a fully atomistic region. The permanent imprint left on the surface of the crystal after retraction is also clearly visible in the deformed mesh.

The computed force vs displacement curve for indentation and retraction is plotted in Fig. 4. As may be seen from this figure, the curve ceases to be monotonic at $6.75 \AA$, at which point an abrupt force drop is observed. This drop is followed by another interval of monotonic increase of the force, in excellent agreement with experimental results [3]. Upon retraction, the force decreases, with the indenter detaching from the crystal surface at a penetration depth of $3 \AA$. This behavior owes to the presence of stable self-equilibrated dislocation structures left in the crystal upon retraction of the indentor,

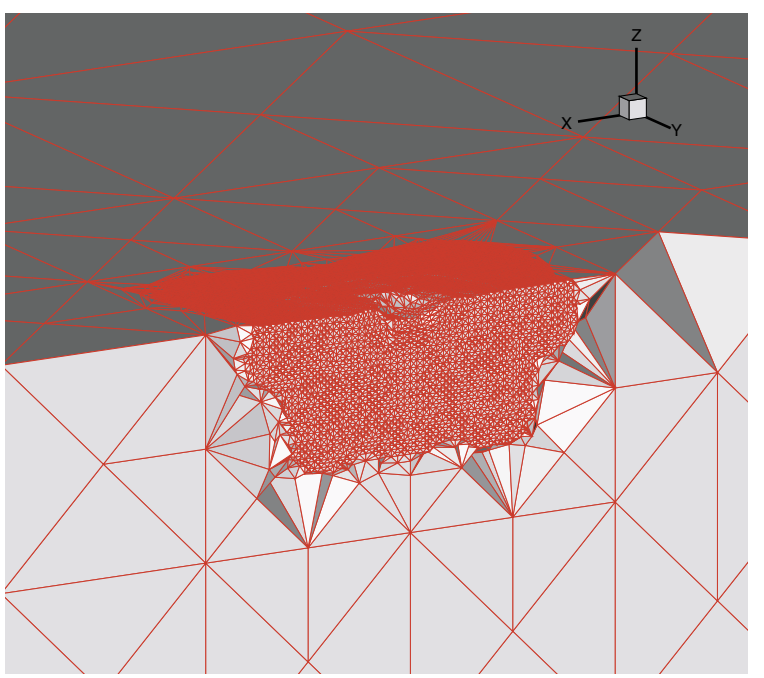

FIG. 3 (color online). Cross section of the computational mesh for the $70 \AA$ indenter at an indentation depth of $9.2 \AA$. 


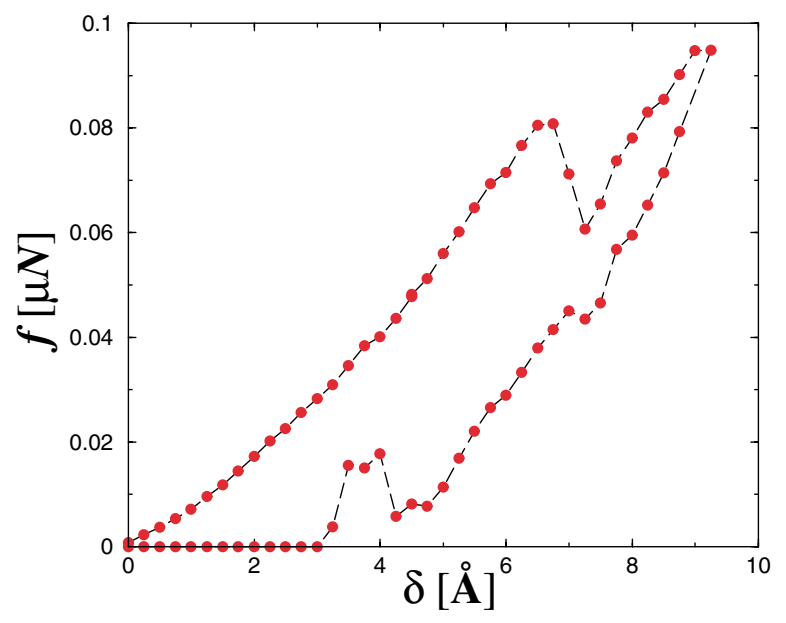

FIG. 4 (color online). Force vs displacement curve for the $70 \AA$ Åradius indenter.

resulting in permanent plastic deformation in the vicinity of the indenter.

The computed dislocation structures for a $700 \AA$-radius indenter at a depth of indentation of $7.5 \AA$ are shown in Fig. 5. A defect structure consisting of a complex array of extended partial dislocation loops is clearly visible in the figure. This dislocation structure differs in notable respects from that which develops under the $70 \AA$ indenter. Thus, the large-indenter dislocation structure is less sharp and more delocalized and contains welldeveloped dislocation loops bounding stacking faults. In addition, the dislocation structure extends over a larger region than in the case of the small indenter, in keeping with the correspondingly larger size of the contact area.

The deformed computational mesh at a depth of penetration of $7.5 \AA$ is shown in Fig. 6. A comparatively

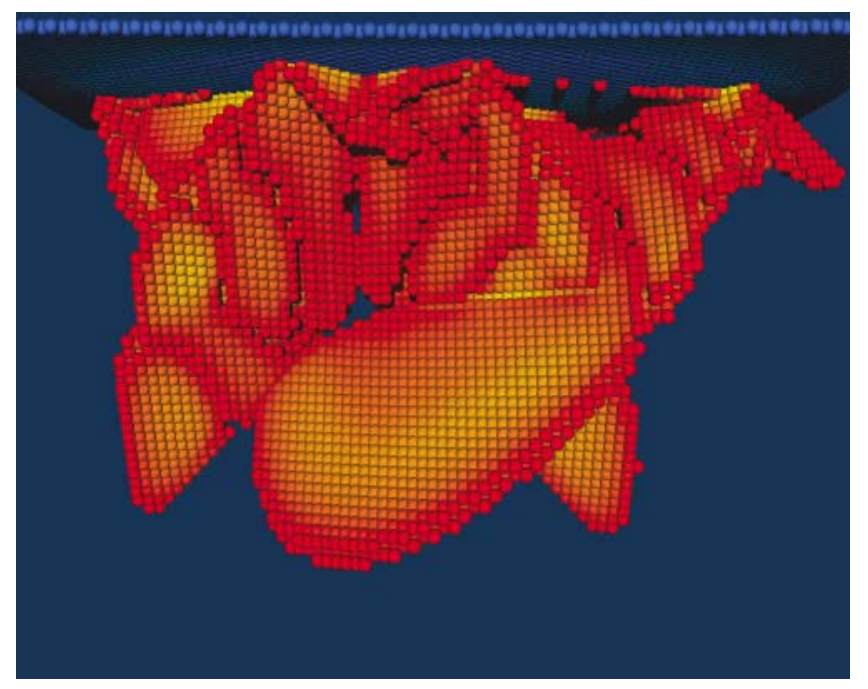

FIG. 5 (color). Dislocation structures for the $700 \AA$ indenter at an indentation depth of $7.5 \AA$.

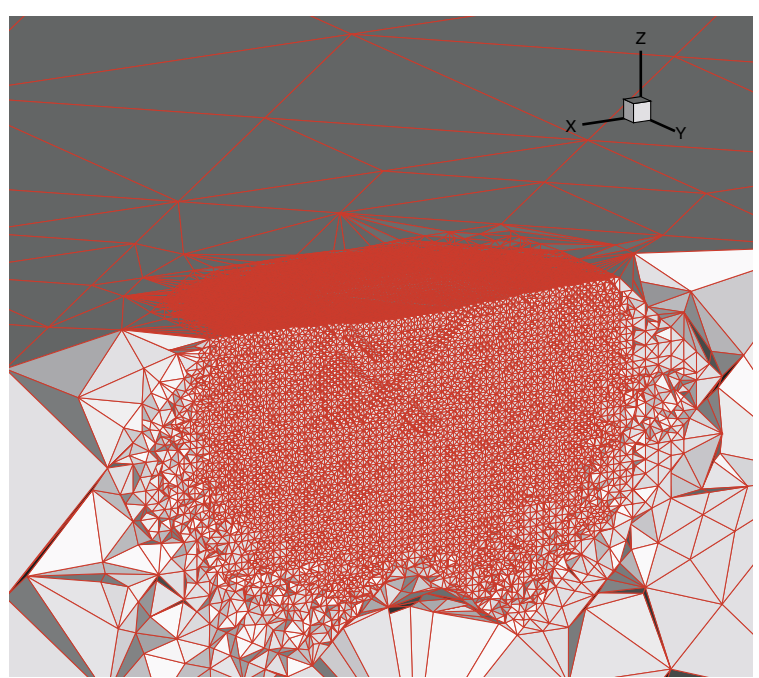

FIG. 6 (color online). Cross section of the computational mesh for the $700 \AA$ indenter at an indentation depth of $7.5 \AA$.

larger atomistic region is needed in the case of the $700 \AA$ indenter in order to encompass the dislocation structure. The larger imprint size left after retraction is also noteworthy.

Figure 7 finally shows the force vs displacement curve for indentation and retraction. As in the $70 \AA$ indenter case, the curve rises during indentation and upon retraction reduces to zero at the indentation depth of $3 \AA$. However, the curve differs from that corresponding to the $70 \AA$ indenter in one important respect, namely, the absence of force drops during indentation, despite profuse dislocation activity. The absence of force drops may be attributed to the large contact area, which averages out the local traction fluctuations introduced by the dislocations.

These observations immediately call into question the correlation between dislocation nucleation events and

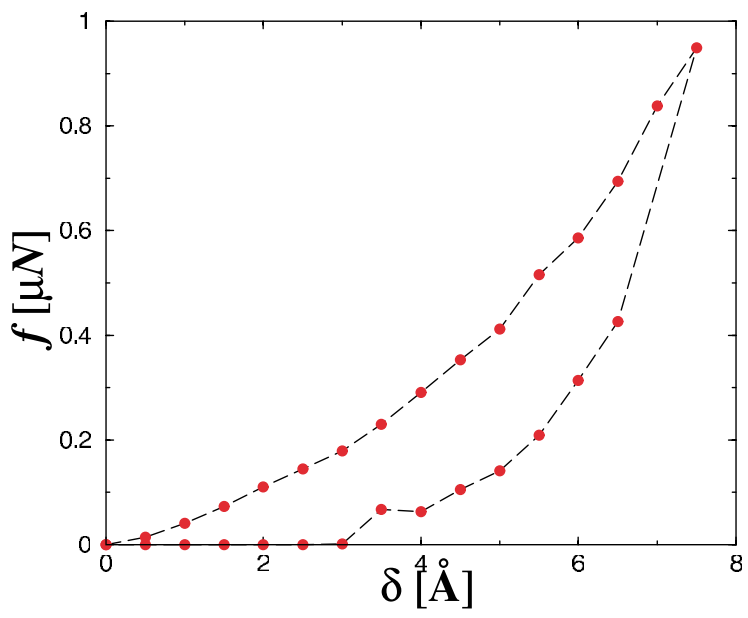

FIG. 7 (color online). Force vs displacement curve for the $700 \AA$ A-radius indenter. 
drops in the force-displacement curve. Specifically, for indenters in the size range actually used in experiment, dislocation activity takes place well in advance of the first force drop, in line with the findings of Kramer et al. [9]. Conversely, the first force drop probably occurs as a result of a dislocation avalanche involving large numbers of previously nucleated dislocations.

It has also been suggested that the onset of the plastic deformation is marked by the deviation of the force curve from the profile obtained by the application of the Hertzian theory of elastic contact. In the investigations of single crystal $\mathrm{Au}(111)$ nanoindentation reported by Kiely et al. [2], this deviation takes the form of abrupt force drops. By contrast, the experimental $\mathrm{Au}(111)$ force curves measured by Michalske and Houston [8] and Kiely et al. [4] do not exhibit any ostensible force drops as they depart from the Hertzian relationship. In these studies, the initial plastic yield is reported to take place at indentation depths in the 70-80 Аे range, or more than an order of magnitude in excess of the values predicted by our calculations.

In conclusion, the indenter force is not a reliable indicator of the onset of dislocation activity and plastic deformation for indenter sizes in the experimental range.

Support from the DOE through Caltech's ASCI/ASAP Center for the Simulation of the Dynamic Response of Solids is gratefully acknowledged.

[1] A. Gouldstone, K. Van Vliet, and S. Suresh, Nature (London) 411, 656 (2001).

[2] J. Kiely, R. Hwang, and J. Houston, Phys. Rev. Lett. 81, 4424 (1998).

[3] J. Kiely and J. Houston, Phys. Rev. B 57, 12588 (1998).

[4] J. Kiely, K. Jarausch, J. Houston, and P. Russell, J. Mater. Res. 14, 2219 (1999).

[5] K. Jarausch, J. Kiely, J. Houston, and P. Russell, J. Mater. Res. 15, 1693 (2000).
[6] D. Bahr and W. Gerberich, Metall. Mater. Trans. A 27, 3793 (1996).

[7] D. Bahr, D. Kramer, and W. Gerberich, Acta Mater. 46, 3605 (1998).

[8] T. Michalske and J. Houston, Acta Mater. 46, 391 (1998).

[9] D. Kramer, K. Yoder, and W. Gerberich, Philos. Mag. A 81, 2033 (2001).

[10] J. Belak, D. Boercker, and I. Stowers, MRS Bull. 18, 55 (1993).

[11] J. Kallman, W. Hoover, C. Hoover, A. Degroot, S. Lee, and F. Wooten, Phys. Rev. B 47, 7705 (1993).

[12] R. Perez, M. Payne, and A. Simpson, Phys. Rev. Lett. 75, 4748 (1995).

[13] C. Kelchner, S. Plimpton, and J. Hamilton, Phys. Rev. B 58, 11085 (1998).

[14] P. Lomdahl, D. Beazley, S. Zhou, and B. Holian, Radiat. Eff. Defects Solids 142, 1 (1997).

[15] S. J. Zhou, D. M. Beazley, P. S. Lomdahl, and B. L. Holian, Phys. Rev. Lett. 78, 479 (1997).

[16] S. Zhou, P. Lomdahl, A. Voter, and B. Holian, Eng. Fract. Mech. 61, 173 (1998).

[17] S. Zhou, D. Preston, and F. Louchet, Acta Mater. 47, 2695 (1999).

[18] K. Kadau, P. Entel, T. Germann, P. Lomdahl, and B. Holian, J. Phys. IV (France) 11, 17 (2001).

[19] P. Vashishta, M. Bachlechner, A. Nakano, T. Campbell, R. Kalia, S. Kodiyalam, S. Ogata, F. Shimojo, and P. Walsh, Appl. Surf. Sci. 182, 258 (2001).

[20] J. Zimmerman, C. Kelchner, P. Klein, J. Hamilton, and S. Foiles, Phys. Rev. Lett. 87, 165507 (2001).

[21] E. Tadmor, R. Phillips, and M. Ortiz, Langmuir 12, 4529 (1996a).

[22] E. Tadmor, M. Ortiz, and R. Phillips, Philos. Mag. A 73, 1529 (1996).

[23] J. Knap and M. Ortiz, J. Mech. Phys. Solids 49, 1899 (2001).

[24] R. A. Johnson, Phys. Rev. B 37, 3924 (1988).

[25] R. A. Johnson, Phys. Rev. B 39, 12554 (1989).

[26] W. H. Press, W. T. Vetterling, S. A. Teukolsky, and B. P. Flannery, Numerical Recipes in $\mathrm{C}++$ : The Art of Scientific Computing (Cambridge University Press, Cambridge, 2002), 2nd ed. 